\title{
Updating multipoint simulations using the ensemble Kalman filter
}

\author{
L.Y. Hu ${ }^{1}$, Y. Zhao ${ }^{2}$, Y. Liu ${ }^{3}$, C. Scheepens ${ }^{4}$ and A. Bouchard ${ }^{5}$
}

\begin{abstract}
In the last two decades, the multipoint simulation (MPS) method has been developed and increasingly used for building complex geological facies models that are conditioned to geological and geophysical data. In the meantime, the ensemble Kalman filter (EnKF) approach has been developed and recognized as a promising way for assimilating dynamic production data into reservoir models. So far, the EnKF approach is proven efficient for updating continuous model parameters that have a linear statistical relation with the flow responses. It remains challenging to extend the EnKF approach to updating complex geological facies models generated by MPS, while preserving their geological and statistical consistency.

In this paper, we introduce a new method for parameterizing geostatistical reservoir models generated by MPS. It is mathematically proven that updating these parameters during a history matching process does not compromise the hard data conditioning and the geological and statistical consistency of the reservoir model defined by the training image and other information including global facies proportions, trend maps etc. This method is an alternative to the gradual deformation method but has an enlarged search space for covering possible solutions. Based on the above parameterization, we present two algorithms of using EnKF approach to update multipoint simulations to dynamic data. We also present encouraging results of using the above methodology to condition a sector model of a fluvial reservoir to dynamic data.
\end{abstract}

\footnotetext{
${ }^{1}$ ConocoPhillips, 600 North Dairy Ashford, P.O. Box 2197, Houston, TX 77252-2197, U.S.A. lin.hu@conocophillips.com

${ }^{2}$ ConocoPhillips, 600 North Dairy Ashford, P.O. Box 2197, Houston, TX 77252-2197, U.S.A. yong.zhao@conocophillips.com

${ }^{3}$ ConocoPhillips, 600 North Dairy Ashford, P.O. Box 2197, Houston, TX 77252-2197, U.S.A. yongshe.liu@ conocophillips.com

${ }^{4}$ ConocoPhillips, 600 North Dairy Ashford, P.O. Box 2197, Houston, TX 77252-2197, U.S.A. claude.scheepens@conocophillips.com

${ }^{5}$ ConocoPhillips, 600 North Dairy Ashford, P.O. Box 2197, Houston, TX 77252-2197, U.S.A. andre.bouchard@conocophillips.com
} 


\section{Introduction}

In the last two decades, the multipoint simulation (MPS) method has been developed and increasingly used for building complex geological facies models that are conditioned to geological and geophysical data (Guardiano and Srivastava, 1993; Strebelle, 2000). Unlike traditional geostatistical simulations based on random function models, a multipoint geostatistical simulation does not require the explicit definition of a random function. It directly utilizes empirical multivariate distributions inferred from training images. This confers on MPS method a potential applicability to any geological environment, provided that there is a training image representative of the geological heterogeneity and that the essential features of this training image can be characterized by statistics defined on a limited point configuration (i.e., template). Hu and Chugunova (2008) provide a comprehensive review of the MPS for reservoir modeling.

In the meantime, the ensemble Kalman filter (EnKF) approach has been developed and recognized as a promising way for assimilating dynamic production data into reservoir models (Evensen, 1994). Aanonsen et al. (2009) give a comprehensive review of the EnKF in petroleum engineering. The EnKF approach is proven efficient for updating continuous model parameters that have a linear statistical relation with the flow responses. Liu and Oliver (2005) first extend the EnKF approach to a truncated Gaussian facies model by using the underlying continuous Gaussian variables as model parameters. The EnKF approach is also used for updating Gaussian mixture models (Dovera and Della Rossa, 2007; Sun et al., 2009). Moreover, several methods are introduced in the literature for representing MPS facies models using continuous parameters (e.g., Moreno and Aanonsen, 2007; Jafarpour and McLaughlin, 2007; Sarma et al., 2008). However, it is not mathematically proven that updating these parameters will preserve the geological and statistical consistency of the MPS facies models defined by training images and other given structure parameters.

Recently, Zhou et al. (2011) propose, at each data assimilation time step, to apply a normal score transformation, independently, to each component of the state vectors, and to update the transformed state vectors. Their method can actually preserve the univariate non-Gaussian distribution of the MPS model. But from the theoretical point of view, it is unclear whether the method can preserve the higher order spatial features of the MPS model in the general case. Jafarpour and Khodabakhshi (2011) propose, at each data assimilation time step, to update the ensemble of log-permeability fields whose mean field is used to derive a facies probability field, then to use the MPS algorithm to generate a new ensemble of log-permeability fields conditioned to the facies probability field for the next data assimilation time step. With this method, the dynamic data are actually used as a soft constraint for generating MPS realizations.

In this paper, we propose a different parameterization method to represent the MPS facies models with a set of continuous parameters. This is motivated by the 
fact that changing or optimizing these parameters will not compromise the geological and statistical consistency of the MPS facies models. Then, we associate the above parameterization method with the EnKF approach for dynamic data integration. This association extends the applicability of the EnKF to complex geological facies models generated by MPS. We also present results of using the above method to update a sector model of channelized reservoir built by multipoint simulation.

\section{Parameterization of multipoint simulations}

\subsection{Sequential multipoint simulation}

We consider the commonly used sequential algorithm for multipoint simulation. The sequential simulation algorithm requires defining an order or path according to which the points in the simulation field are visited and simulated one after another. This simulation path can be regular or irregular, and it can be fixed prior to generating realizations. For each point of the simulation field, we calculate the probability distribution of the facies property conditioned to the facies at the data points and at the already simulated points. This conditional distribution is based on the multipoint statistics from the training image. Then, we draw a facies property from this conditional distribution using a uniform random number between 0 and 1 . There is a uniform random number for each simulation point. The ensemble of uniform numbers constitutes a spatially independent uniform field (or vector).

Therefore, like the general case of sequential simulation, building geomodels using the sequential MPS algorithm can also be considered as a mapping that transforms a field of independent and uniformly distributed random numbers into a field of geological properties distributed according to a training image and a set of structural parameters (e.g., global facies proportions) (Hu et al., 2001). Namely, given a training image $T i$ and a set of structural parameters $S$, a geomodel $M$ is a function of a spatially independent uniform random field $U$, i.e.

$$
M_{T i, S}(U)
$$

Hereafter, we simply call $U$ uniform field or uniform white noise. From the above definition, it is clear that any realization drawn from the uniform field $U$ gives a MPS realization that is consistent with the training image and the specified structural parameters.

Updating realizations of the MPS model (1) consists of changing the training image (geological scenarios) $T i$, modifying the structural parameters $S$ and/or updating the uniform field $U$. In general, there are only a few geological 
scenarios (training images) and a limited number of structural parameters (global facies proportions, etc.) to be considered. However, the uniform field has a huge number of components that equals the number of cells of the geomodel. Moreover, to preserve the spatial consistency of the geomodel, these components cannot be arbitrarily modified and instead must remain a uniform white noise.

\subsection{Parameterization}

We propose a parameterization of the uniform field that allows us to reduce the huge number of parameters of the geomodel (1), and at the same time to preserve the geomodel spatial consistency when modifying these parameters during the subsequent uncertainty analysis and history matching processes.

Let $U_{1}, U_{2}, \ldots, U_{n}$ be a series of uniform fields that are independent between each others. We define a new random field $V$ as a linear combination of $U_{1}, U_{2}, \ldots, U_{n}$

$$
V\left(r_{1}, r_{2}, \ldots, r_{n}\right)=\sum_{i=1}^{n} r_{i} U_{i}
$$

where $r_{i} \in(-\infty,+\infty)$ are the combination coefficients. In practice, it is more convenient to use the tangent transformation

$$
r_{i}=\operatorname{tg}\left(\frac{\pi \rho_{i}}{2}\right)
$$

and to use $\rho_{i} \in(-1,1)$ as parameters instead of $r_{i}$.

The mean of $V$ equals $\sum_{i} r_{i} / 2$, and the variance of $V$ equals $\sum_{i} r_{i}^{2} / 12$. However, unlike the linear combination of Gaussian random fields that remains Gaussian, the linear combination of uniform fields is no longer uniform, except for the trivial cases where $r_{i}=1$ and $r_{j}=0$ for $j=1, \ldots, i-1, i+1, \ldots, n$. Figure 1 shows the histogram of a realization of a linear combination of 100 uniform fields, which is clearly not uniform.

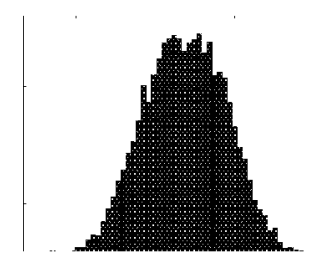

Figure 1: Histogram of a linear combination of 100 uniform fields. 
Nevertheless, we can transform $V$ into a uniform field $U$ by the uniform score transformation (also known as uniform anamorphosis)

$$
U(\rho)=F[V(\rho)]
$$

where $\rho$ stands for the parameter vector $\left(\rho_{1}, \rho_{2}, \ldots, \rho_{n}\right)$. Like the normal score transformation (Gaussian anamorphosis), the uniform score transformation is performed component per component for the vector $V$ and the transformation function is entirely determined by the marginal cumulative distribution function (cdf) of $V$. Except for the case of small number $n$, it is very tedious to derive the analytical expression of this cdf. In practice, we resort to the empirical cdf of $V$ to calculate $U$ (Figure 2).

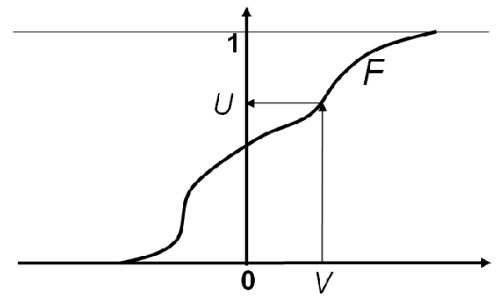

Figure 2: Uniform score transformation.

Now, we replace the uniform field $U$ in the definition (1) by the combined uniform field $U(\rho)$ defined by (2). Therefore, given $n$ independent realizations of the uniform field, the geomodel (1) becomes a function of $n$ coefficients $\rho_{1}, \rho_{2}, \ldots, \rho_{n}$ of their combination: $M_{T i, S}\left(\rho_{1}, \rho_{2}, \ldots, \rho_{n}\right)$.

These combination coefficients can be arbitrarily modified without compromising the spatial consistency of the MPS realization. Thus they can be updated using any optimization method during the subsequent uncertainty analysis and history matching processes.

The above parameterization method is an alternative to the gradual deformation method (Hu, 2000, Le Ravalec et al., 2000). It differs from the gradual deformation in that the uniform fields are directly combined instead of converted to Gaussian random fields for their combinations. Another feature of the method is that, unlike the gradual deformation method, the sum of the squares of the combination coefficients $r_{i}$ is not limited to 1 . Actually, the range of $r_{i}$ is $(-\infty$, $\infty)$, allowing an enlarged realization space to cover possible solutions. 


\section{EnKF applied to multipoint simulations}

We consider two algorithms of using the ensemble Kalman filter (EnKF) approach to update geomodels generated by MPS. The first algorithm is to update directly the uniform field $U$, and the second algorithm is to update the combination coefficients $\rho_{1}, \rho_{1}, \ldots, \rho_{n}$. Figure 3 is a schematic representation of the two workflows based on the above two algorithms. The first algorithm involves as many parameters as the number of cells of the geomodel, whereas the second algorithm involves a user defined number of parameters. To simplify the presentation, we consider, for both algorithms, the geological scenario (training image) is fixed, although different scenarios can be considered in practice. Besides, we also fix the structure parameters, although they can be updated simultaneously with the uniform field or with the combination coefficients $\rho_{1}, \rho_{2}, \ldots, \rho_{n}$.

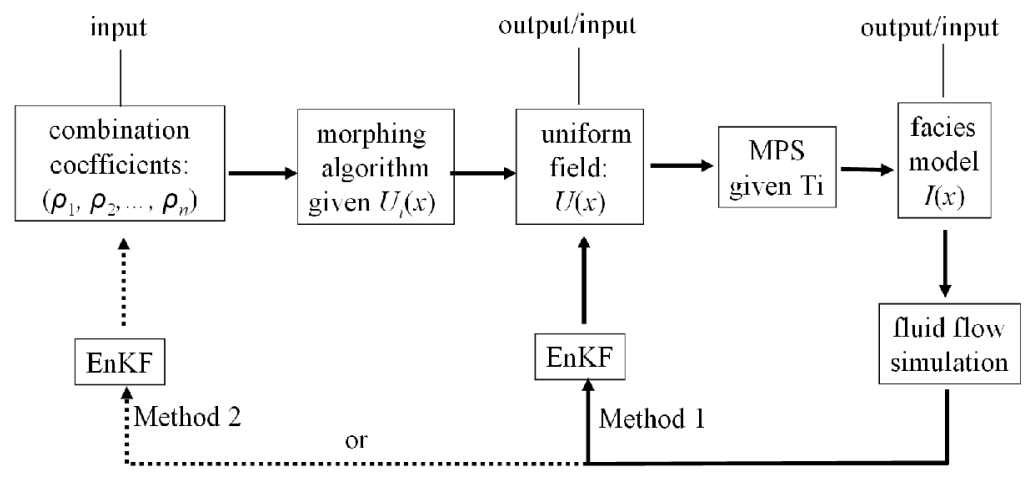

Figure 3: Two workflows of updating MPS to dynamic data using EnKF.

Note that the idea of using the uniform field in definition (1) as parameters in inverse modeling is also proposed by Capilla et al. (1999). However, in their inverse modeling algorithm, the uniform field (called probability field in their paper) is perturbed by a spatially correlated field. Thus, the resulting probability field is no longer a uniform white noise and, the subsequent realization is no longer consistent with the definition (1) where $U$ must be a uniform white noise. In later work on using the uniform field as parameters in inverse modeling, the uniform field is parameterized and updated by using the gradual deformation method (Hu et al., 2001; Capilla and Llopis-Albert, 2009), thus remains a uniform white noise during the updating process. The following inverse modeling algorithms based on EnKF also preserve the characteristics of the uniform white noise, thus the characteristics of the geomodel by definition (1). 


\subsection{EnKF applied to the uniform random field}

Consider first using the EnKF approach to update directly the uniform field. The algorithm is as follows:

Initiation:

1. Set $t=0$ for the initial time step;

2. Generate an initial ensemble of $n$ uniform fields: $U_{i}^{t}(x), i=1,2, \ldots, n$. $x \in D$, where $D$ stands for the reservoir field or grid;

3. For all uniform fields $U_{i}^{t}(x)$, compute their corresponding standard Gaussian fields using the inverse standard Gaussian cdf: $Y_{i}^{t}(x)=G^{-1}\left[U_{i}^{t}(x)\right], i=1,2, \ldots, n ;$

Iteration:

4. Build the reservoir model $M_{i}^{t}(x)$ for each uniform field $U_{i}^{t}(x)$, $i=1,2, \ldots, n$, according to definition (1);

5. Perform flow simulation for each $M_{i}^{t}(x)$ between time steps 0 and $t+1$

6. Update the ensemble of Gaussian fields $\left\{Y_{i}^{t}(x), i=1,2, \ldots, n\right\}$ using EnKF from time step $t$ to time step $t+1:\left\{Y_{i}^{t+1}(x), i=1,2, \ldots, n\right\}$;

7. For all the updated Gaussian fields $Y_{i}^{t+1}(x)$, compute, component per component, their corresponding uniform fields using the standard Gaussian cdf: $U_{i}^{t+1}(x)=G\left[\frac{Y_{i}^{t+1}(x)}{\sigma_{i}^{t+1}}\right], i=1,2, \ldots, n$, where $\sigma_{i}^{t+1}$ stands for the (empirical) standard deviation of the Gaussian field $Y_{i}^{t+1}(x)$;

8. Set $t=t+1$; go to 4 until the last time step.

Note that, at each time step of the sequential updating procedure, only the geomodel realizations are updated by EnKF, their corresponding pressure and saturation fields are obtained by performing fluid flow simulations from the initial time step $t=0$. This avoids the inconsistency between the updated geomodel realizations and their corresponding flow responses, but obviously requires much more CPU time for flow simulation.

As pointed out by Aanonsen et al. (2009), a Gaussian field updated by EnKF is a linear combination of the initial Gaussian fields, thus remains Gaussian. The EnKF shares this feature with the gradual deformation method. The difference is that, when using the above EnKF algorithm, the model parameters are the components of the Gaussian fields instead of their combination coefficients with 
the gradual deformation method. However, the Gaussian realizations updated by EnKF are not necessarily standard Gaussian. They need to be standardized before their back transform to uniform fields at step 7. The fact that $U_{i}^{t+1}(x)$ for $x \in D$ remains a uniform white noise guarantees the subsequent consistency of the geomodel realization, for instance in teams of the geometric features defined by the training image and the given structural parameters.

\subsection{EnKF applied to the combination coefficients}

Consider now using the EnKF approach to update the coefficients of combining a set of uniform fields. The number of combination coefficients is user defined. In general, we use a small number of parameters (combination coefficients) with respect to the number of cells $N$ of the geomodel. Thus, we have a reduced flexibility for modifying the geomodel and for matching the dynamic data. The algorithm is as follows:

Initiation:

1. Generate an ensemble of $n$ uniform fields: $U_{i}(x), i=1,2, \ldots, n$, $x \in D$ the reservoir field (grid);

2. Set $t=0$ for the initial time step;

3. Generate an initial ensemble of $k$ uniform vectors $\rho_{l}^{t}=\left(\rho_{l, 1}^{t}, \rho_{l, 2}^{t}, \ldots, \rho_{l, n}^{t}\right), \quad l=1,2, \ldots, k$, all components of these vectors are uniform numbers between -1 and 1 ;

4. For all vectors $\rho_{l}^{t}, l=1,2, \ldots, k$, compute their corresponding standard Gaussian $\quad Y_{l}^{t}=\left(Y_{l, 1}^{t}, Y_{l, 2}^{t}, \ldots, Y_{l, n}^{t}\right) \quad$ wectors: with $Y_{l, i}^{t}=G^{-1}\left(0.5+0.5 \rho_{l, i}^{t}\right), i=1,2, \ldots, n$

Iteration:

5. For each vector of combination coefficients $\rho_{l}^{t}=\left(\rho_{l, 1}^{t}, \rho_{l, 2}^{t}, \ldots, \rho_{l, n}^{t}\right)$, perform the linear combination: $\sum_{i=1}^{n} \operatorname{tg}\left(\frac{\pi \rho_{l, i}^{t}}{2}\right) U_{i}(x)$, then compute its corresponding uniform field $U_{l}^{t}(x)$ by the uniform score transformation;

6. Build the reservoir model $M_{l}^{t}(x)$ for each $U_{l}^{t}(x), l=1,2, \ldots, k$, according to definition (1); 
7. Perform flow simulation for each $M_{l}^{t}(x)$ between time steps 0 and $t+1$

8. Update the ensemble of Gaussian vectors $\left\{Y_{l}^{t}, l=1,2, \ldots, k\right\}$ using EnKF from time step $t$ to time step $t+1:\left\{Y_{l}^{t+1}, l=1,2, \ldots, k\right\}$;

9. For all the updated Gaussian vectors $Y_{l}^{t+1}, l=1,2, \ldots, k$, compute their corresponding vectors of combination coefficients: $\rho_{l}^{t+1}=\left(\rho_{l, 1}^{t+1}, \rho_{l, 2}^{t+1}, \ldots, \rho_{l, n}^{t+1}\right)$ with $\rho_{l, i}^{t+1}=2 G\left(Y_{l, i}^{t+1}\right)-1, i=1,2, \ldots, n ;$

10. Set $t=t+1$; go to step 5 until the last time step.

Note that, unlike the first algorithm, the ensemble size of the above EnKF algorithm is denoted $k$ instead of $n$ that is used for denoting the number of uniform fields in both algorithms. Like the first algorithm, only the geomodel realizations are updated by EnKF, their corresponding pressure and saturation fields are obtained by performing fluid flow simulations from the initial time step $t=0$.

Likewise, each updated vector $Y_{l}^{t+1}=\left(Y_{l, 1}^{t+1}, Y_{l, 2}^{t+1}, \ldots, Y_{l, n}^{t+1}\right)$ remains Gaussian, but not necessarily standard Gaussian (its variance may differ from 1). However, unlike the first algorithm, these Gaussian vectors $Y_{l}^{t+1}, l=1,2, \ldots, k$ do not need to be standardized before their back transform to uniform vectors at step 9 . This is because the geomodel realizations following steps 5 and 6 are geologically and statistically consistent for any values of the combination coefficients.

\section{Examples}

A test model of $50 \times 50$ cells is created with the multipoint simulation algorithm SNESIM (Strebelle, 2000). The model is based on a commonly used training image of $250 \times 250$ cells representing a fluvial channel system (Figure 4 ).

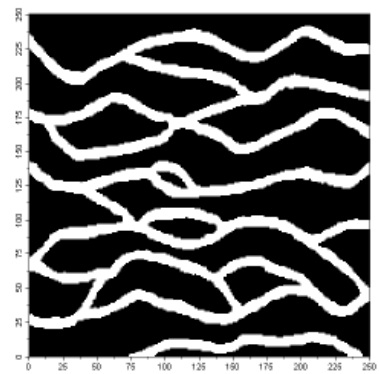

Figure 4: Training image of $250 \times 250$ cells representing a fluvial channel system. 
For the purpose of the test, a first random realization is generated and considered to represent a sector of a hydrocarbon reservoir (Figure 5). The realization is called the target realization and the objective of the test is to demonstrate that the proposed EnKF algorithms can be used to assimilate simulated dynamic data from the target realization in a history matching process.

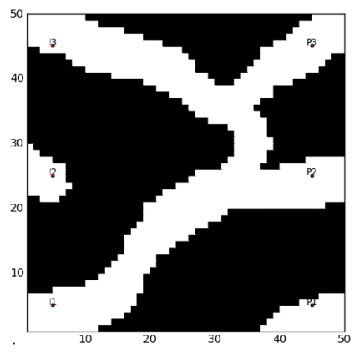

Figure 5: Target realization.

There are three water injection wells on the left side of the sector model and three production wells on the right side of the sector model. All the six wells are in the channel facies. This constitutes the hard data conditioning of all model realizations. The dynamic data set is composed of the water rate at the three injectors, the water rate and oil rate at the three producers.

An ensemble of 100 initial random realizations based on the above model is then generated. Two of them are represented in Figure 6, together with a so called channel probability map, which is a graphic representation of the probability of finding the channel facies in a particular location and is in essence an average of the 100 initial realizations.

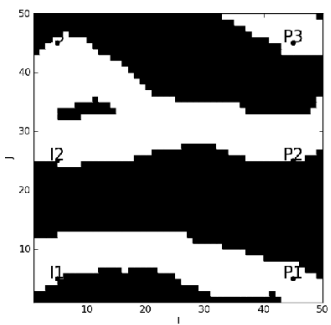

a) Realization \#40

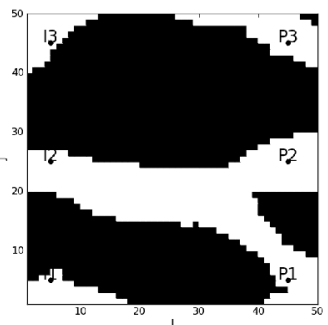

b) Realization \#100

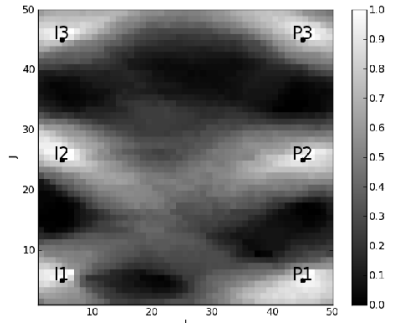

c) Average of 100 realizations

Figure 6: Two initial realizations (a, b) among 100 and the average of the 100 initial realizations representing the initial channel probability map (c). 


\subsection{EnKF applied to the uniform field}

We first use the algorithm described in section 3.1 where the facies model is parameterized by a uniform field. The EnKF is applied to assimilate the dynamic data set from the target realization to update the initial ensemble. The number of parameters to update is actually the number of cells of the facies model, i.e. 2500.

An ensemble of 100 updated realizations is generated, and two of them are shown in Figure 7, together with the channel probability map based on the updated ensemble. It may easily be seen that the updated probability map (Figure 7c) captures much better the inter-well connectivity of the target (Figure 5) than does the initial probability map (Figure 6c). In practice, one may use the updated average facies map (updated probability map) for well planning instead of using an updated individual realization.

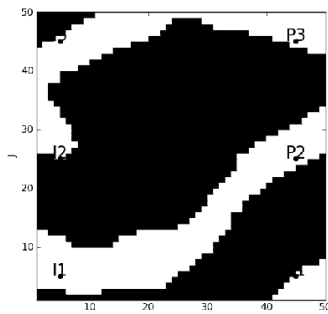

a) Realization \#40

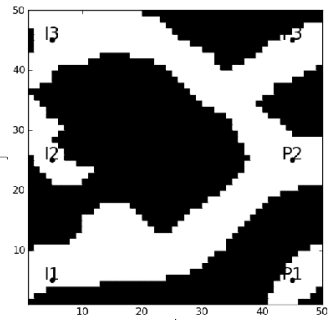

b) Realization \#10

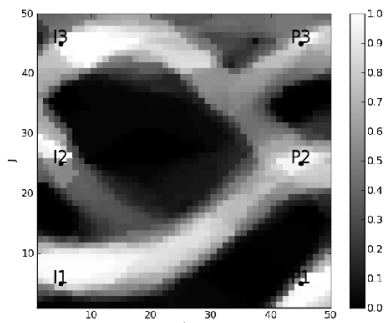

c) Average of 100 realizations

Figure 7: Two updated realizations (a, b) among 100 and the average of the 100 updated realizations representing the updated channel probability map (c).

In contrast to the average dynamic response of the initial ensemble, the average dynamic response of the updated ensemble is very close to the simulated dynamic data from the target realization (Figures 8, 9, 10). Therefore, one may use the average production curves of the updated ensemble for production forecasting instead of using the individual production curves of the updated ensemble of realizations. Note also that, after assimilating the dynamic data, the gap between the p10 and p90 curves is much reduced for the water rate of injectors 1 and 2, and for both water and oil rates of producer 1, but remains almost unchanged for the water rate of injector 3 and for both water and oil rates of producers 2 and 3 . 

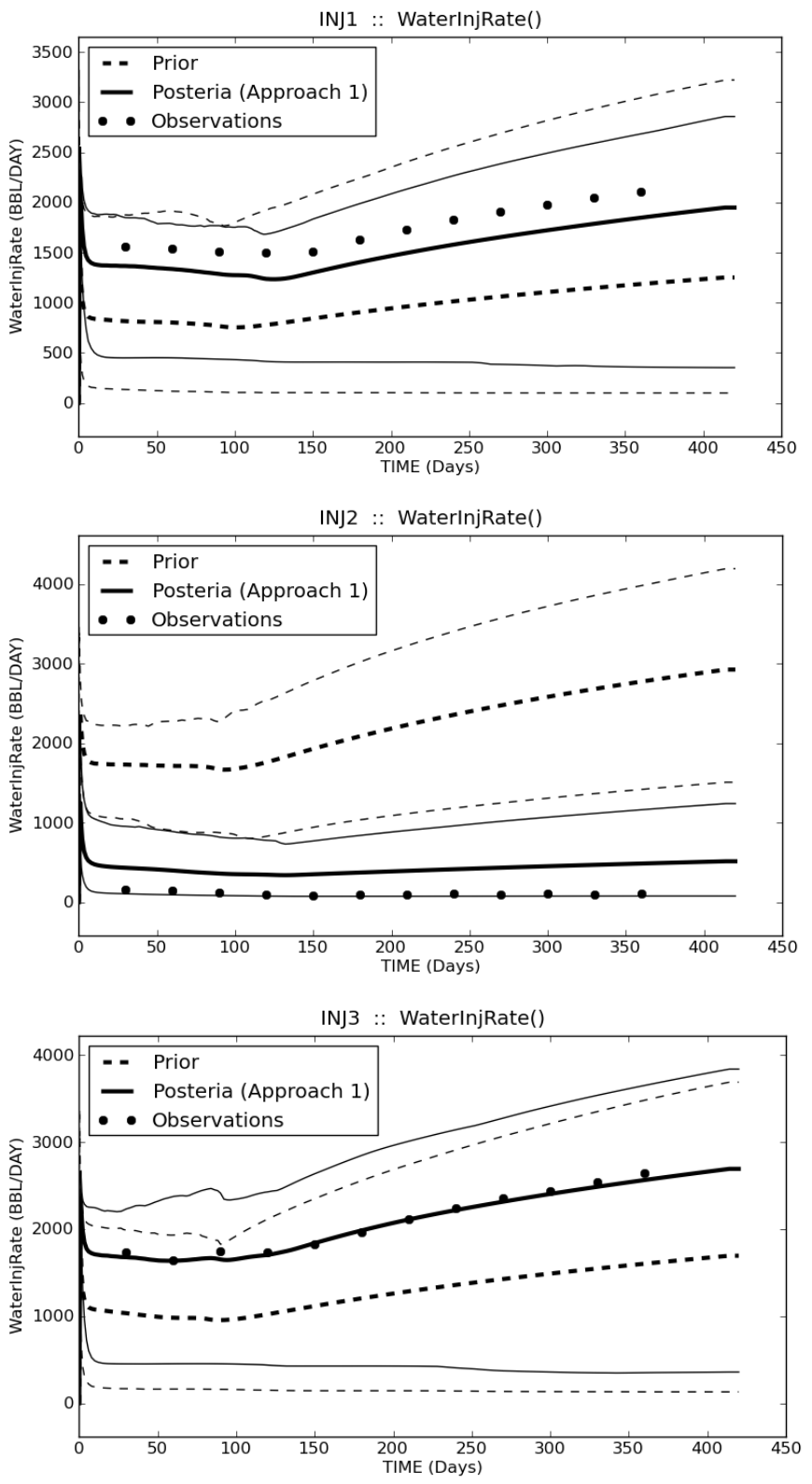

Figure 8: Injector water rates from the target realization (dots), the initial ensemble (thick dashed line representing the mean, thin dashed lines the p10 and p90) and the updated ensemble (thick solid line representing the mean, thin solid lines the p10 and p90). 

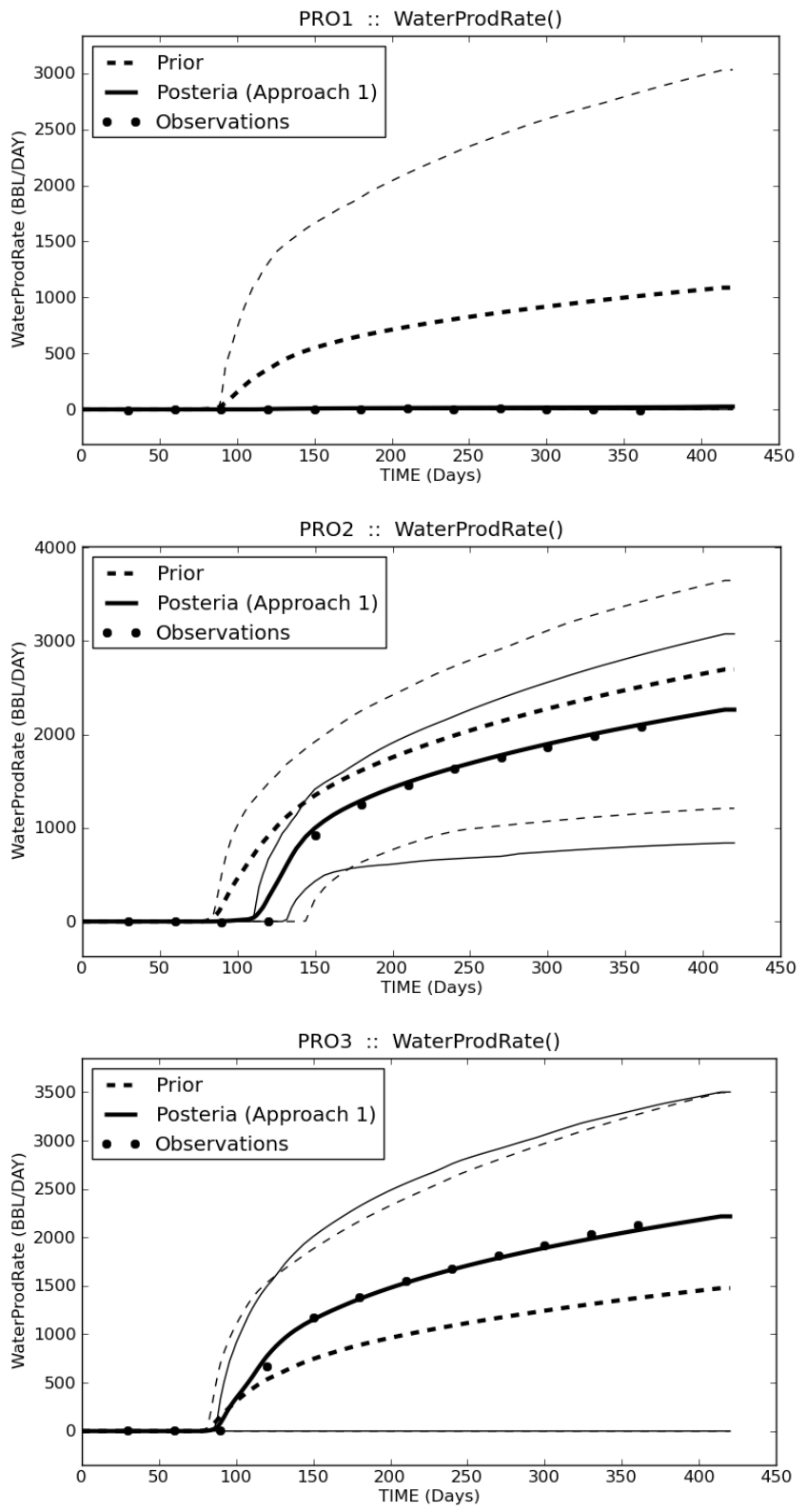

Figure 9: Producer water rates from the target realization (dots), the initial ensemble (thick dashed line representing the mean, thin dashed lines the p10 and p90) and the updated ensemble (thick solid line representing the mean, thin solid lines the p10 and p90). 

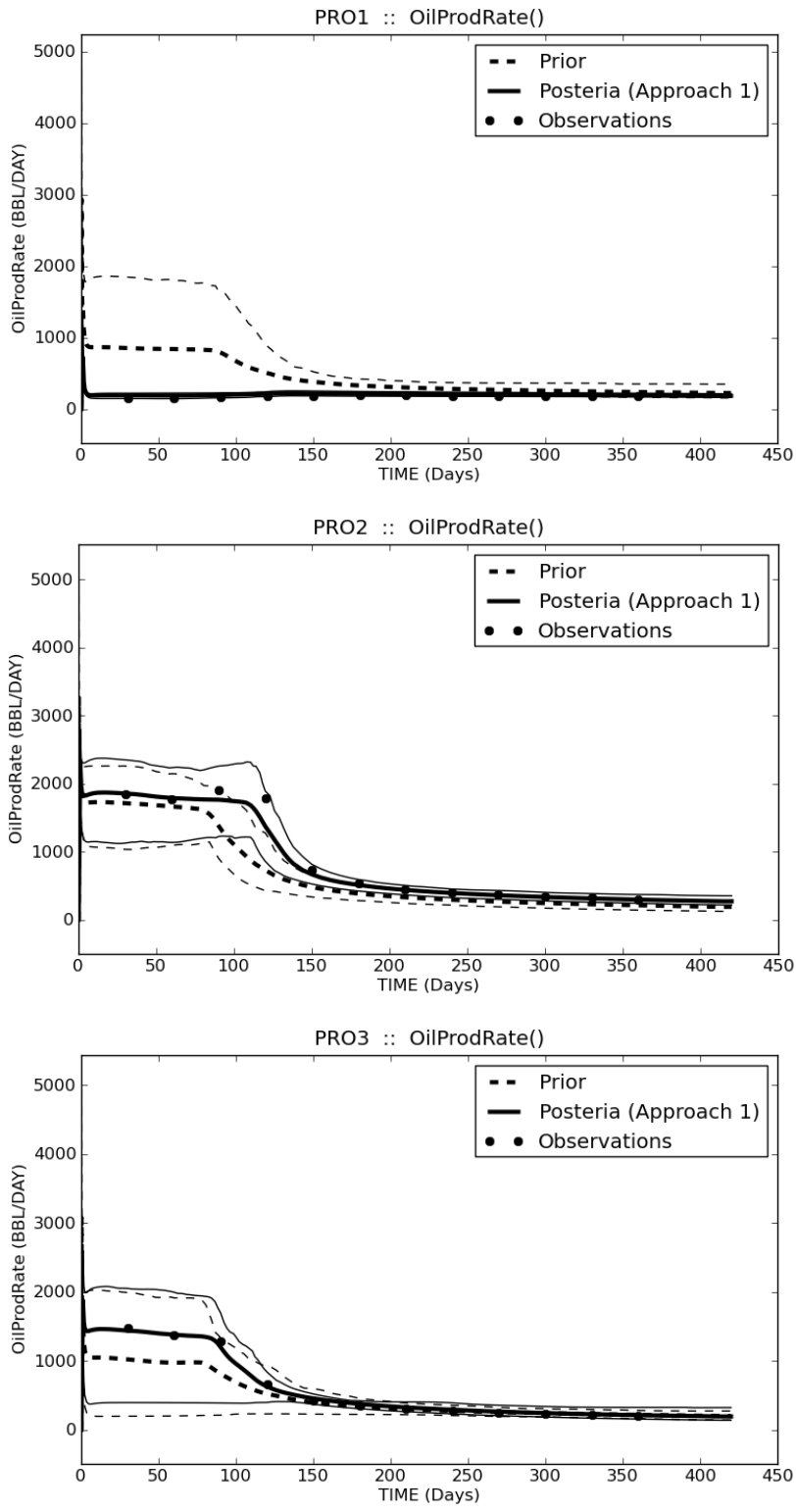

Figure 10: Producer oil rates from the target realization (dots), the initial ensemble (thick dashed line representing the mean, thin dashed lines the p10 and p90) and the updated ensemble (thick solid line representing the mean, thin solid lines the p10 and p90). 


\subsection{EnKF applied to the combination coefficients}

Now we use the algorithm described in section 3.2 where the facies model is parameterized by a set of combination coefficients. The EnKF is applied to assimilate the dynamic data from the target realization by updating the coefficients of combining the initial 100 realizations of the previous example. Thus in this example, the EnKF deals with 100 model parameters instead of 2500 parameters as in the previous example.

We also use an ensemble of 100 members in this example, although the ensemble size can be different from the number of parameters. An ensemble of 100 initial sets of combination coefficients is generated, and this leads to an ensemble of 100 initial realizations of the geomodel. After applying the EnKF algorithm in section 3.2, an ensemble of 100 updated realizations is generated. Two of them are shown in Figure 11, together with the channel probability map based on the updated ensemble of 100 realizations. In general, this probability map (Figure 11c) is improved with respect to the initial probability map (Figure $6 \mathrm{c}$ ), but it is not as good as in the previous example when updating directly the uniform field. This is basically due to the reduction of the number of parameters from 2500 to 100 , thus the reduction of the flexibility to modify the facies model. Moreover, the combination coefficients are global parameters in the sense that their modification involves a global change of the facies model.

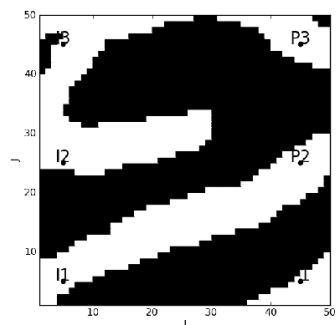

a) Realization \#40

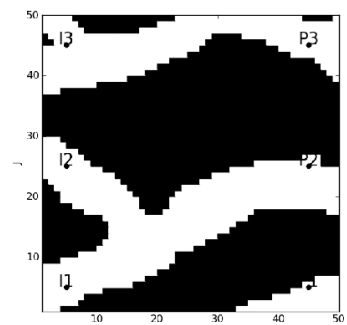

b) Realization \#100

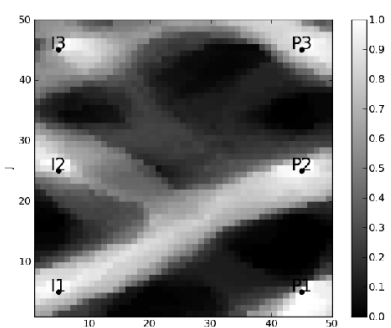

c) Average of 100 realizations

Figure 11: Two updated realizations (a, b) among 100 and the average of the 100 updated realizations representing the updated channel probability map.

There are two ways of increasing the flexibility of the model updating. One way is to increase the number of realizations to combine. This increases the number of combination coefficients, thus the flexibility to modify the model. Note that even if the number of combination coefficients is increased, we may use an ensemble size smaller than the number of parameters just like in the previous example with 2500 but with an ensemble size of 100 . Another way of increasing the number of parameters is to divide the reservoir field into a certain number of regions, and by updating the combination coefficients in these regions 
simultaneously but separately. Both ways are investigated for updating a continuous property model with gradual deformation parameterization by Heidari et al. (2011) and are proven effective. We believe this will also be the case of using the parameterization presented in this paper for updating the MPS facies model.

\section{Conclusions and discussions}

In this paper, we introduced a new method for parameterizing geostatistical reservoir models generated by multipoint simulation. It is mathematically proven that updating these parameters during a history matching process will not compromise the hard data conditioning and the geological and statistical consistency of the reservoir model defined by the training image and other information including global facies proportions, trend maps etc. This method is an alternative to the gradual deformation method but has an enlarged search space to cover possible solutions. Like the gradual deformation method, the method is not limited to the MPS and can also be used for parameterizing any geostatistical reservoir model.

Based on the above parameterization, we presented two algorithms of using the EnKF approach to update MPS simulations to dynamic data. One algorithm applies the EnKF directly to all components of the uniform field and the other algorithm applies the EnKF to the coefficients of combination of uniform fields. The first algorithm involves as many parameters as the number of cells of the geomodel, thus provides a lot of flexibility for updating the ensemble. As for the second algorithm, the number of parameters is user defined, and in general much smaller than the number of cells of the geomodel.

For both algorithms, all updated realizations from the two test examples are, by construction, consistent with the prior geological and statistical data. However, due to the nonlinearity between the model parameters and the dynamic responses, many individual realizations from the updated ensemble do not sufficiently match the dynamic data. Nevertheless, in the example of direct updating of the uniform field, the average dynamic responses of the updated ensemble are very close to the dynamic data, and the average facies map (facies probability map) of the updated ensemble provides a very good probabilistic representation of the target facies distribution. In practice, these average solutions are very useful for production forecasting and for well planning. In the example of updating the combination coefficients, the efficiency of the EnKF is reduced due to the relatively small number of parameters used and an increased nonlinearity between the model parameters and the dynamic responses.

In this work, we explored the basic EnKF method that is optimal for assimilating data linearly related to the model parameters. To further improve the dynamic data match, one may explore the combination of the MPS 
parameterization proposed in this paper with a nonlinear EnKF method, e.g., the EnKF using distance in kernel space proposed by Park et al. (2008). One may also reparameterize the flow responses as proposed by Chen et al. (2009) to reduce the non-Gaussianity of the EnKF state vector. These are directions of our future work.

Another important issue is whether the ensemble of realizations updated by the proposed methods can correctly represent the remaining uncertainty about the reservoir heterogeneity. This issue is beyond the scope of the present paper and, it can only be addressed when we are able to build an ensemble of updated realizations that are both geologically realistic and sufficiently history-matched.

\section{References}

1. Aanonsen, S.I., Nævdal, G., Oliver, D.S., Reynolds, A.C. and Valles, B., 2009. The ensemble Kalman filter in reservoir engineering - A review. SPE Journal, 14(3), 393-412.

2. Capilla, J. E., Rodrigo, J. and Gomez-Hernandez, J. J., 1999. Simulation of non-Gaussian transmissivity fields honoring piezometric data and integrating soft and secondary information. Mathematical Geology, 31(7), 907-927.

3. Capilla, J. E. and Llopis-Albert, C., 2009. Gradual conditioning of nonGaussian transmissivity fields to flow and mass transport data: 1. Theory. Journal of Hydrology, 371(2009), 66-74.

4. Chen, Y., Oliver, D.S. and Zhang, D., 2009. Data assimilation for nonlinear problems by ensemble Kalman filter with reparameterization. Journal of Petroleum Science and Engineering, 66(2009), 1-14.

5. Dovera, L. and Della Rossa, E., 2007. Ensemble Kalman filter for Gaussian mixture models. Paper A16 presented at the 2007 EAGE/SPE Petroleum Geostatistics Conference, 10-14 September 2007, Cascais, Portugal.

6. Evensen, G., 1994. Sequential data assimilation with a non-linear quasigeostrophic model using Monte Carlo methods to forecast error statistics. Journal of Geophysical Research, 10(99), 143-162.

7. Guardiano, F. and Srivastava, M., 1993. Multivariate geostatistics: Beyond bivariate moments. In: Soares, A., ed., Geostatistics Troia'92, vol. 1, 133-144, Kluwer Acad. Pub.

8. Heidari, L., Gervais, V., Le Ravalec, M. and Wackernagel, H., 2011. Historymatching of petroleum reservoir models by the ensemble Kalman filter and parameterization methods. to be published.

9. Hu, L.Y., 2000. Gradual deformation and iterative calibration of Gaussianrelated stochastic models. Mathematical Geology, 32(1), 87-108.

10. $\mathrm{Hu}$, L.Y., Blanc, G. and Noetinger, B., 2001. Gradual deformation and iterative calibration of sequential stochastic simulations. Mathematical Geology, 33(4), 475-489. 
11. Hu, L.Y. and Chugunova, T., 2008. Multiple-point geostatistics for modeling subsurface heterogeneity: A comprehensive review. Water Resources Research, 44, W11413, doi: 10.1029/2008WR006993.

12. Jafarpour, B. and McLaughlin, D.B., 2007. History matching with an ensemble Kalman filter and discrete cosine parameterization. SPE 108761 presented at the 2007 SPE Annual Technical Conference and Exhibition, 1114 November 2007, Anaheim, CA.

13. Jafarpour, B. and Khodabakhshi, M., 2011. A probability conditioning method (PCM) for nonlinear flow data integration into multipoint statistical facies simulation. Mathematical Geosciences, 43(2011), 133-164.

14. Le Ravalec, M., Noetinger, B. and Hu, L.Y., 2000. The FFT moving average (FFT-MA) generator: an efficient numerical method for generating and conditioning Gaussian simulation. Mathematical Geology, 32(6), 701-723.

15. Liu, N. and Oliver, D.S., 2005. Ensemble Kalman filter for automatic history matching of geologic facies. Journal of Petroleum Science and Engineering, 47(3-4), 147-161.

16. Moreno, D. and Aanonsen, S., 2007. Stochastic facies modeling using the level set method. Paper A18 presented at the 2007 EAGE/SPE Petroleum Geostatistics Conference, 10-14 September 2007, Cascais, Portugal.

17. Park, K., Scheidt, C. and Caers, J., 2008. Simultaneous conditioning of multiple non-Gaussian geostatistical models to highly nonlinear data using distances in kernel space. In: Ortiz, J.M. and Emery, X., eds, Proceedings of the 8th International Geostatistics Congress, Santiago, Chile, 247-256.

18. Sarma, P., Durlofsky, L. and Aziz, K., 2008. Kernel principal component analysis for efficient, differentiable parameterization of multipoint geostatistics. Mathematical Geosciences, 40(1), 3-32

19. Strebelle, S., 2000. Sequential simulation drawing structures from training images. Ph.D. Dissertation, Petroleum Engineering Department, Stanford University, USA.

20. Sun, A.Y., Morris, A.P. and Mohanty, S., 2009. Sequential updating of multimodal hydrogeologic parameter fields using localization and clustering techniques. Water Resources Research, 45, W07424.

21. Zhou, H., Gomez-Hernandez, J.J., Hendricks Franssen, H.-J. and Li, L., 2011. An approach to handling non-Gaussianity of parameters and state variables in ensemble Kalman filtering. Advances in Water Resources, 34(2011), 844864. 